\title{
Fluctuating Bond Aggregation: a Model for Chemical Gel Formation
}

\author{
Rémi Jullien and Anwar Hasmy \\ Laboratoire de Science des Matériaux Vitreux, Université Montpellier II, Place Eugène Bataillon, \\ 34095 Montpellier, France
}

(August 6, 2018)

\begin{abstract}
The Diffusion-Limited Cluster-Cluster Aggregation (DLCA) model is modified by including cluster deformations using the bond fluctuation algorithm. From $3 d$ computer simulations, it is shown that, below a given threshold value $c_{g}$ of the volumic fraction $c$, the realization of all intra-aggregate bonding possibilities prevents the formation of a gelling network. For $c>c_{g}$, the sol-gel transition occurs at a time $t_{g}$ which, in contrast to DLCA, doesnot diverge with the box size. Several results are reported including small angle scattering curves and possible applications are discussed.
\end{abstract}

PACS numbers: 61.43.Bn, 61.43.Hv, 82.70.Gg. 
If the sol-gel process is widely used to design several materials of practical interest, the physics of the polymerisation (aggregation) mechanism that leads to gel formation is far to be completely understood, even in the simple case of chemical gels where the aggregation of $f>2$ functional monomers is due to polycondensation reactions [1]. Several complementary theories have been proposed. The kinetic approach [2], which is based on the use of the Smoluchowski equation, has the advantage to well describe the sol-gel transition as due to the appearence of an infinite aggregate at a given gel time $t_{g}$. But it doesnot give a precise description of the gel structure as all the geometrical aspects are hidden in the kernel of the equation. On the opposite, the de Gennes and Stauffer approach [3, [1], in which the sol-gel process is viewed as a bond percolation transition [1], can account for the remarkable self-similar (fractal) properties of the gel structure but cannot describe all the irreversible kinetical features, as the percolation theory is restricted to thermodynamical equilibrium. A great hope was born twelve years ago with the introduction of kinetic aggregation models [5] since they were able to describe both kinetical and geometrical aspects. In particular the diffusion-limited cluster-cluster aggregation (DLCA) algorithm [6,7] simulates directly the aggregation process on a computer by letting particles, as well as aggregates of particles, diffusing in a box (with periodic boundary conditions (PBC)) and sticking irreversibly when they meet. The DLCA model, as well as some of its variants [8], were often used to describe the formation of chemical gels [1,8]. However, as first pointed out by Kolb and Herrmann [9], a major problem arises as $t_{g}$ tends to infinity in the thermodynamical limit of an infinitely large box. Correlatively, the concentration threshold $c_{g}$, above which gelation can take place, vanishes.

In this letter, we show that allowing cluster deformations during aggregation removes these difficulties. Such an extension also provides a more realistic description of chemical gel formation, since, in experiments, intra-aggregate motions always exist up to some degree, due to free-rotations, bond angle deformations etc... We report on a $3 d$ simulation of an aggregation process of $f$-functional subunits, in which, in addition to rigid motions of clusters, some internal movements are introduced with a tuning flexibility parameter $F$. This is made 
possible by extending the efficient Bond Fluctuation method [10,11]. Such kinetic rules are essentially chosen for the sake of efficiency and it is clear that any real dynamics would proceed differently, but we are convinced that the results obtained are sufficiently general to have a wide range of applications. We find that, for a volumic fraction (concentration) $c$ smaller than a given threshold $c_{g}$, the saturation of all intra-cluster bounding possibilities prevents the formation of a gelling network while for $c>c_{g}$, gelation occurs at a given time $t_{g}$ which does not tend to infinity with the box size. Here we give the most significant results and we show how they can account for some experiments. A more detailed report will be published later.

Our numerical simulation considers a cubic lattice of unit parameter limited to a cubic box of edge length $L$. The moving particles are considered as hard cubes of edge length 2 whose centers can jump by one unit from site to site (taking care of PBC). Bonding vectors between particle centers are restricted to a set of 108 possibilities: $[ \pm 2,0,0],[ \pm 2, \pm 1,0],[ \pm 2, \pm 1, \pm 1],[ \pm 2, \pm 2, \pm 1],[ \pm 3,0,0],[ \pm 3, \pm 1,0]$ (and permutations). The directions $[ \pm 2, \pm 2,0]$ (and permutations) are forbidden to avoid bond crossing [11]. At $t=0, N$ unbonded particles are randomly disposed in the box, avoiding overlaps (the volumic fraction $c$ is related to $N$ by $c=8 N / L^{3}$ ). Then, we use an iterative procedure in which aggregates (= particles connected by bonds), are built. At a given iteration, an aggregate of $n$ particles (or a single particle if $n=1$ ) is picked up at random with probability $P_{n}$ and one decides, either to move it globally and rigidly by a random unit jump, or to move one of its particles (chosen at random), with probabilities $Q_{n}$ and $1-Q_{n}$, respectively. If the chosen motion is compatible with bond restrictions and hard core conditions, it is performed and one proceeds with the next iteration. In the other case, any overlapping attempt is detected before pursuing with the next iteration. If there is one and if the corresponding particles are not saturated (i. e. they both have less than $f$ bonds), a new bond is created between them (if there are several possibilities, one is chosen at random). Therefore, in this model, a new bond is formed only when one particle tries to overlap another one, as in the off-lattice DLCA model [12]. Another restriction has been added which is to forbid the cre- 
ation of bonds triangles. This artificial (and rather technical) condition has been introduced to prevent the formation of tetrahedra which, due to our bond restrictions, cannot move on large distances by successive jumps of single particles. The following expressions have been chosen for $P_{n}$ and $Q_{n}$ :

$$
P_{n}=\frac{1+F n}{1+F} n^{\alpha} / \sum \frac{1+F n}{1+F} n^{\alpha} ; \quad Q_{n}=\frac{1}{1+F n}
$$

where the sum runs over all the clusters and $\alpha$ is a kinetic exponent. Knowing that, if only single particle jumps are allowed per unit time, the mobility of an aggregate of $n$ particles varies as $1 / n$ (as for linear polymers [11]), such expressions insure, for any $F$, a cluster mobility proportional to $n^{\alpha}$, as in DLCA. It is known [8] that $\alpha$ should be taken equal to $-1 / D$, where $D$ is the cluster fractal dimension, to insure a cluster diffusivity varying as the inverse of the radius. In all the calculations presented here we have taken $\alpha=-0.5$, in agreement with the resulting fractal dimension, of order 2 (we have checked that varying $\alpha$ around the chosen value doesnot affect too much the results). As a consequence of (1), the physical time $t$ is calculated by adding $\delta t=1 / \sum \frac{1+F n}{1+F} n^{\alpha}$ at each iteration.

We have checked numerically that all the known properties (in particular $D \simeq 1.8$ ) of the $3 d$ DLCA model are well recovered for $F=0, f>2$, and in addition, due to our rule for bond creation, we get no loop, as in the off-lattice case [12]. Here, to illustrate the influence of cluster flexibility, we shall present some numerical results for a typical large $F$ value $(F=125)$. As the functionality $f$ increases the dynamics is lowered due to bond constraints. Therefore, we have taken $f=3$, which is the lowest value able to produce a gel ( $f=2$ corresponds to an interesting problem of flexible chains aggregation which has been previously studied with a different method [13]). The existence of a concentration threshold $c_{g} \simeq 0.055$ is illustrated qualitatively in fig. 1 and quantitatively in fig.2. For $c<c_{g}$, even after waiting a very long time (the figures correspond to $t=10^{5} \times N$ ), no gel is obtained and we get a collection of clusters, a lot of them being saturated (i.e. all their particles have $f$-bonds). In this concentration range, we have plotted their mean radius of gyration $R$ as a function of $c$. A more and more pronounced divergence at $c_{g}$ is observed as $L$ increases. 
For $c>c_{g}$, we observe that one cluster becomes self-connected via the PBC at a given time $t_{g}$, i.e. it appears a true infinite cluster when repeating the box by translations of $L$ in the three directions (note that such condition defines a gel better than the one previously used in DLCA [9:12]). The mean values for $t_{g}$, almost independent on $L$ (in contrast to DLCA), also exhibit a quasi-divergence at $c_{g}$ which is quite well fitted by $t_{g} \sim\left(c-c_{g}\right)^{-2}$ (In the case of $R$ we need larger size calculations to be able to estimate any critical exponent). We have done a few other calculations with different $F$ values and we have observed that $c_{g}$ increases monotically with $F$ and seems to saturate as $F \rightarrow \infty$ to a limiting value $c_{g \infty}$ only slightly larger to the one reported here for $F=125$, while the characteristics of the transition at $c_{g}$ remain the same. Moreover preliminary calculations with $f=4$ indicate that $c_{g \infty}$ is smaller than with $f=3$.

In figure 3 we report on numerical results for the Small-Angle Neutron Scattering (SANS) curves of the gel structure obtained for $c>_{\sim} c_{g}$, in the case $f=3, F=125$. The structure factor $S(q)$, which the Fourier transform of the particle centers distances, has been calculated by:

$$
S(q)=<\left|\sum_{\vec{r}}(\rho(\vec{r})-\bar{\rho}) e^{i \vec{q} \cdot \vec{r}}\right|^{2}>=\sum_{\overrightarrow{r_{1}}} \sum_{\overrightarrow{r_{2}}}\left(\rho\left(\overrightarrow{r_{1}}\right) \rho\left(\overrightarrow{r_{2}}\right)-\bar{\rho}^{2}\right) \frac{\sin q\left|\overrightarrow{r_{1}}-\overrightarrow{r_{2}}\right|}{q\left|\overrightarrow{r_{1}}-\overrightarrow{r_{2}}\right|}
$$

where $V=L^{3}, \bar{\rho}=N / V,<\ldots>$ designs the average over the $\vec{q}$ directions, $\rho(\vec{r})=1$ if the site $\vec{r}$ is occupied by a particle center and $\rho(\vec{r})=0$ otherwise. As explained in [12], it is essential, when one deals with a gel, to substract $\bar{\rho}$ to avoid finite size artifacts due to the box. To compare with experiments, it should be remembered that the SANS intensity curve $I(q)$ might be obtained from $S(q)$ by multiplying by a form factor $P(q)$ characterizing the shape of the particles. This has not been done here due to the crudeness of the model which considers cubic particles connected by massless bonds. The log-log curves exhibit the same qualitative shape as for DLCA [12], i.e. a maximum at low- $q$, which is shifted to larger $q$ as the concentration increases, an intermediate fractal linear regime, followed by damped oscillations (characteristic of the short range order between particles [14]) at larger $q$-values. However, for $c>_{\sim} c_{g}$, the absolute value of the fractal slope $(\simeq 2.3)$ is significantly 
larger than in DLCA and decreases slowly as $c$ increases. Since the fractal dimension of the aggregates forming the gel is very close to 2 (as seen on the mass-radius curve in inset and also checked by box-counting calculations), the slope of $I(q)$ is not equal to $D$ when $c$ is close to $c_{g}$. This is a strong indication that the scaling function characterizing the size-distribution of clusters should exhibit a large size power law tail for $c>_{\sim} c_{g}, t<_{\sim} t_{g}$, in contrast to the DLCA case where it is known to decrease exponentially [8]. Despite numerical uncertainties, a preliminary calculation of the cluster size distribution confirms this hypothesis. A power law size distribution is not surprising since, in that parameters range, the system exhibits all the characteristics of criticality. Note also that, for $c>_{\sim} c_{g}, t<_{\sim} t_{g}$, only a few bonds per cluster are available to build the gel, and therefore the efficiency of collisions is very weak as in Chemically Limited (also called Reaction Limited) Cluster-Cluster Aggregation for which it is known that $D \simeq 2$ [8].

In this letter we have shown that including cluster deformations alters significantly the properties of cluster-cluster aggregation: a true sol-gel transition is obtained. More calculations will be performed to make more precise the roles of the different parameters, to study more quantitatively the scaling properties close to $c_{g}$ and $t_{g}$, and to compare with percolation theory. We are convinced that, due to the general character of its results, the present model can be applied to several experimental situations. Here we would like to discuss a possible application to silica aerogels. While base catalysed aerogels, which result from the colloidal aggregation of mesoscopic silica particles, are known to form quite rigid structures, well described by DLCA [12], the neutral aerogels are more likely made of a flexible polymeric network, since, from SANS experiments, the lower cut-off of the fractal regime is found to be of atomic scale [15]. The slope of the SANS curve is larger (about 2.3) and a fractal dimension of $\sim 2.5$ was reported by fitting with an analytical formula (valid for a single fractal cluster) [15]. Such a larger slope might very well be explained by the present model since it is also known, from thermoporometry experiments [16], that the mesoscopic pore size distribution is larger than in base catalysed aerogels, in agreement with a strong cluster size polydispersity. We are now suggesting some more experiments to test such a 
conjecture.

We benefited from discussions with Marie Foret and Thierry Woignier. One of us (A.H.) would like to acknowledge support from CONICIT (Venezuela). Numerical calculations were done with the help of the computers of the CNUSC (Centre Universitaire Sud de Calcul). 


\section{REFERENCES}

[1] C. Brinker and G. W. Scherer, Sol-Gel Science: The Physics and Chemistry of Sol-Gel Processing, Academic Press, New York 1990.

[2] M. H. Ernst, in Fundamental Problems in Statistical Mechanics, ed. E. Cohen, 6, 329, Elsevier, New York 1985.

[3] P. G. de Gennes, Scaling Concepts in Polymer Science, Cornell Univ. Press, Ithaca 1979.

[4] D. Stauffer, Introduction to percolation theory, Phys. Rep., 54, 1, 1979.

[5] F. Family and D. P. Landau, Kinetics of Aggregation and Gelation, North-Holland, 1984.

[6] P. Meakin, Phys. Rev. Lett., 51, 1119, 1983.

[7] M. Kolb, R. Botet and R. Jullien, Phys. Rev. Lett., 51, 1123, 1983.

[8] R. Jullien and R. Botet, Aggregation and Fractal Aggregates, World Scientific, 1987.

[9] M. Kolb and H. Hermann, J. Phys. A, 18, L435, 1985.

[10] J. Carmesin and K. Kremer, Macromolecules, 21, 2819, 1988.

[11] W. Paul, K. Binder, D. W. Heermann and K. Kremer, J. Chem. Phys., 95, 7726, 1991.

[12] A. Hasmy, E. Anglaret, M. Foret, J. Pelous and R. Jullien, Phys. Rev. B, 50, 1305, 1994.

[13] L. Turban and J. M. Debierre, J. Phys. A, 20, 4457, 1987.

[14] A. Hasmy, M. Foret, J. Pelous and R. Jullien, Phys. Rev. B 48, 9345, 1993.

[15] R. Vacher, T. Woignier, J. Pelous and E. Courtens, Phys. Rev. B, 37, 6500, 1988.

[16] T. Woignier, J. Phalippou, J. F. Quinson, M. Pauthe, M. Reppellin-Lacroix and G. W. Scherer, J. of Sol-Gel Science and Techn. , 2, 277, 1994. 


\section{FIGURES}

FIG. 1. Typical configurations obtained with $f=3, L=120, F=125$. Cases (a) and (b) correspond to $c=0.02$ and $c=0.06$, respectively. In case (a) the aggregation process was run up to $t=10^{5} \times N$ while in case (b) it has been stopped after obtaining a single cluster. Bonds between particles connected via periodic boundary conditions are not shown

FIG. 2. Mean radius of gyration $R$ (circles) and gel time $t_{g}$ (squares) as a function of $c$ for $f=3, F=125$ and two box sizes $L=60$ (open symbols) and 120 (filled symbols). The data result from an average over 50 and 10 independent simulations for $L=60$ and 120, respectively.

FIG. 3. SANS curves $S(q)$ calculated for $f=3, L=60, F=125$ and three values of $c$ : $c=0.055,0.065,0.075$. For comparison the curve corresponding to $c=0.065, F=0$ is shown (dashed line). In inset the mass-size curves of the aggregates for $c=0.065 F=125$ (solid line) and $F=0$ (dashed line) are shown. In the figure and inset the curves have been arbitrarily vertically shifted for convenience. 

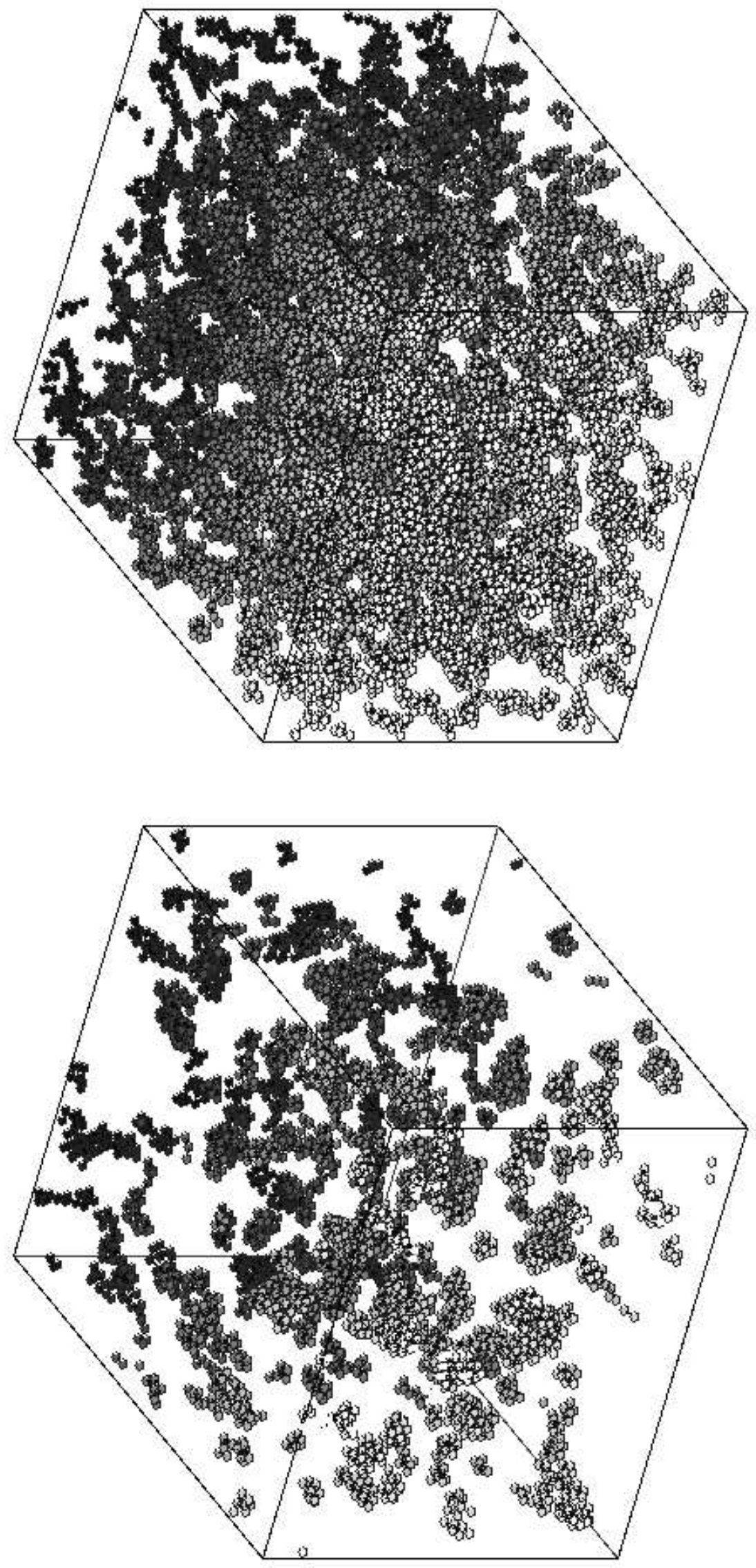







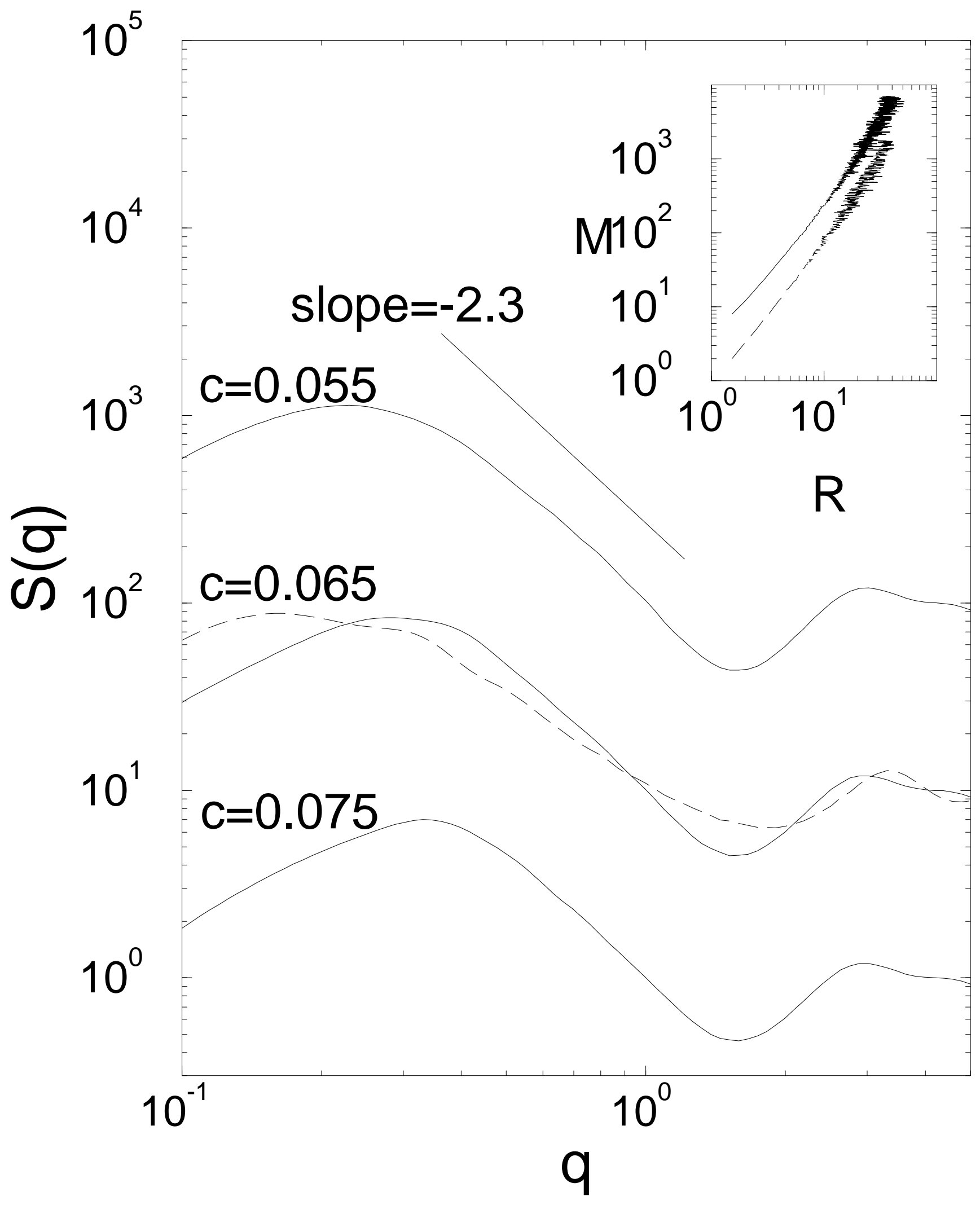

